\title{
THE WKB METHOD FOR CONJUGATE POINTS IN THE VOLUMORPHISM GROUP
}

\author{
STEPHEN C. PRESTON
}

\section{INTRODUCTION}

In this paper, we are interested in the location of conjugate points along a geodesic in the volumorphism group $\mathcal{D}_{\mu}\left(M^{3}\right)$ of a compact three-dimensional manifold $M^{3}$, without boundary. As shown in [?], these are typically pathological, i.e., they can occur in clusters along a geodesic, unlike on finite-dimensional Riemannian manifolds. We give an explicit algorithm for finding them in terms of a certain ordinary differential equation, derived via the WKB-approximation methods of Lifschitz-Hameiri [?] and Friedlander-Vishik [?]. We prove that for a typical geodesic in $\mathcal{D}_{\mu}\left(M^{3}\right)$, there will be pathological conjugate point locations filling up closed intervals in $\mathbb{R}^{+}$; hence typically the zeroes of Jacobi fields on the volumorphism group are dense in intervals.

Interest in the geometry of the volumorphism group dates back to 1966, when Arnold [?] showed that if the volumorphism group is equipped with an $L^{2}$ Riemannian metric, then the geodesics of this metric correspond to Lagrangian solutions of the ideal fluid equations. In particular, the right-translated tangent vector to the geodesic will be an incompressible velocity field satisfying the Euler equation of ideal fluid mechanics. Arnold then computed the Riemannian sectional curvature of this metric in some special cases, showing that it was often negative, and concluded that most fluid flows were likely to be unstable in the Lagrangian sense. In addition, he found some sections of positive curvature, and suggested that the existence of conjugate points would imply that there were some stable perturbations of a fluid flow.

This geometric approach to equations of continuum mechanics has since been used by many authors to find partial differential equations whose solutions represent geodesics on an infinite-dimensional Lie group. Well-known equations arising in this way include the Korteweg-de Vries equation, the Camassa-Holm equation, and the Landau-Lifschitz equation. See for example [?] for an overview. In some cases, this approach only works formally: the geodesic equation resulting may not be a genuine ordinary differential equation on a Banach manifold in any reasonable topology. On the volumorphism group, however, the geodesic equation is very well-behaved in a Sobolev topology: Ebin and Marsden [?] proved in 1970 that if we extend the group of $C^{\infty}$ volumorphisms $\mathcal{D}_{\mu}(M)$ to the space of Sobolev class $H^{s}$ volumorphisms $\mathcal{D}_{\mu}^{s}(M)$, there is a

Date: July 28, 2021.

Some of this work was completed while the author was a Visiting Assistant Professor at Stony Brook University. The author is grateful for their hospitality. 
$C^{\infty}$ Riemannian exponential map defined in some open subset of each tangent space $T_{\mathrm{id}} \mathcal{D}_{\mu}^{s}(M)$. In addition, the differential of the exponential map can be computed in terms of the Jacobi equation in the same way as on a finite-dimensional manifold; Misiołek [?] proved in 1992 that the parallel transport and curvature operators are smooth in the Sobolev topology, so that the Jacobi equation is also an ordinary differential equation. These results allow one to study ideal fluids using standard Picard iteration techniques rather than PDE estimates.

The first examples of conjugate points on the diffeomorphism group were found by Misiołek, who in [?] gave a simple example of a fluid flow on $\mathcal{D}_{\mu}\left(S^{3}\right)$ having a conjugate point, and in [?] gave a more complicated example of a fluid flow on $\mathcal{D}_{\mu}\left(\mathbb{T}^{2}\right)$ having a conjugate point. Arnold and Khesin, in their comprehensive guide to the geometry of the diffeomorphism group [?], asked whether one could expect conjugate points to accumulate along a geodesic, or whether they were isolated as on a finite-dimensional Riemannian manifold. (In general, as shown by Grossmann [?], conjugate points on an infinite-dimensional manifold may cluster together along a geodesic. Grossmann defined monoconjugate and epiconjugate points in terms of the failure of the differential of the exponential map to be injective or surjective, respectively, and showed that the two types need not coincide in infinite-dimensions. In addition, there may be clustering of monoconjugate or epiconjugate points along geodesics.)

This question was answered by Ebin, Misiołek, and the author [?]. We showed that for any two-dimensional manifold without boundary, the exponential map is a nonlinear Fredholm map of index zero. Thus epiconjugate points and monoconjugate points are the same, and furthermore all monoconjugate points have finite order and are isolated along a geodesic. On the other hand, we showed that on the three-dimensional manifold $D^{2} \times S^{1}$ (the solid torus with flat metric), a certain rotational flow has an epiconjugate point that is not monoconjugate. The author [?] extended this result to show that on any three-dimensional manifold, the first conjugate point along any geodesic is pathological: either epiconjugate and not monoconjugate, or monoconjugate of infinite order. That paper used the index form to locate the first conjugate point, and the method was based on finding a simple ordinary differential equation at each point of the manifold whose solutions would give an upper bound for the location of a conjugate point. However, since it relied on the index form rather than the actual Jacobi equation, it could not be used to find the location of any conjugate point other than the first.

In this paper, we approximate the Jacobi equation itself by an ordinary differential equation at each point of the manifold, by working with highly-peaked perturbations of the fluid flow. This technique is well-known as the WKB method, first applied in quantum mechanics. It was first applied to the linearized Euler equation by Bayly [?] in a special case, then more generally by Lifschitz-Hameiri [?] and Friedlander-Vishik [?] in the early 90s. By a result of the author [?], the Jacobi equation can be decomposed into the linearized Euler equation and a simple linearized flow equation; as a result, we can apply the WKB method to the Jacobi equation as well. We do this in Section 
3 to obtain the ordinary differential equation

$$
\pi_{\xi_{o}^{\perp}}\left[\frac{d}{d t}\left(\Lambda\left(t, x_{o}\right) \frac{d \gamma}{d t}\right)+\omega_{o}\left(x_{o}\right) \times \frac{d \gamma}{d t}\right]=0, \quad \gamma(0)=0, \quad\left\langle\gamma(t), \xi_{o}\right\rangle \equiv 0
$$

Here $\Lambda\left(t, x_{o}\right)=D \eta\left(t, x_{o}\right)^{\mathrm{T}} D \eta\left(t, x_{o}\right)$ is the metric deformation of the flow $\eta$ at $x_{o} \in M$, while $\omega_{o}\left(x_{o}\right)=\operatorname{curl} u_{o}\left(x_{o}\right)$ is the initial vorticity at $x_{o}$. The vector $\xi_{o}$ is a fixed unit vector in $T_{x_{o}} M$. We prove that if for some fixed $x_{o} \in M$ and $\xi_{o} \in T_{x_{o}} M$, there is a solution $\gamma(t)$ of (1.1) satisfying $\gamma(0)=0$ and $\gamma(a)=0$, then there is an epiconjugate point in $\mathcal{D}_{\mu}\left(M^{3}\right)$ located at $t=a$.

The freedom to specify any $x_{o}$ and any $\xi_{o} \in T_{x_{o}} M$ implies the existence of many epiconjugate points. In Section 4, we consider at a fixed $x_{o} \in M$ the set of all $a \in \mathbb{R}^{+}$ and $\xi_{o} \in S^{2}$ such that (1.1) has a solution orthogonal to $\xi_{o}$ and vanishing at $t=0$ and $t=a$. By analyzing the equation (1.1) in detail, we show that this set is a collection of two-dimensional surfaces in $\mathbb{R}^{+} \times S^{2}$, which can be expressed as the graph of a function on some open subset of $S^{2}$. From this, we conclude that the epiconjugate point locations form closed nontrivial intervals in $\mathbb{R}^{+}$. As we point out with an example, these intervals frequently extend to infinity.

\section{BACKGROUND}

Our basic object is a compact Riemannian manifold $M$ of dimension $n \geq 2$, without boundary, whose Riemannian metric generates a volume form $\mu$. (For the most part we will assume $M$ is three-dimensional, although this is not necessary.) It is a well-known result of Arnold [?] that formally the geodesic equation on the volumorphism group $\mathcal{D}_{\mu}(M)$, consisting of all $C^{\infty}$ diffeomorphisms $\eta: M \rightarrow M$ with $\eta^{*} \mu=\mu$, where $\mu$ is the Riemannian volume form, is the equation of ideal fluid mechanics in Lagrangian coordinates, when $\mathcal{D}_{\mu}(M)$ is given the $L^{2}$ Riemannian metric

$$
\langle u \circ \eta, v \circ \eta\rangle_{L^{2}}=\int_{M}\langle u, v\rangle \circ \eta \mu=\int_{M}\langle u, v\rangle \mu .
$$

Here the tangent space at $\eta \in \mathcal{D}_{\mu}(M)$ is given by

$$
T_{\eta} \mathcal{D}_{\mu}(M)=\{v \circ \eta \mid \operatorname{div} v=0\} .
$$

The differentials of the left- and right-translations are given by

$$
D L_{\eta}(v)=D \eta(v) \quad \text { and } \quad D R_{\eta}(v)=v \circ \eta .
$$

By equation (2.2), the $L^{2}$ metric is right-invariant under the group action, but not left-invariant unless $\eta$ is an isometry.

Although the above works formally, to treat ordinary differential equations we need a Hilbert manifold structure, which we obtain by enlarging both $\mathcal{D}_{\mu}(M)$ and $T_{\eta} \mathcal{D}_{\mu}(M)$ to the spaces of Sobolev-class $H^{s}$ diffeomorphisms and $H^{s}$ vector fields, with $s>n / 2+1$. Ebin-Marsden established that the geodesic equation for a diffeomorphism $\eta$ is a smooth second-order differential equation on this manifold, so that it has smooth solutions and in particular a smooth exponential map $\exp _{\text {id }}: \Omega \subset T_{\text {id }} \mathcal{D}_{\mu}^{s}(M) \rightarrow \mathcal{D}_{\mu}^{s}(M)$, defined by 
$\exp _{\text {id }}\left(u_{o}\right)=\eta(1)$, where $\eta$ is the geodesic with $\eta(0)=\mathrm{id}$ and $\dot{\eta}(0)=u_{o}$. (By rightinvariance of the metric, it is no loss of generality to assume all geodesics begin at the identity map, which we will do from now on.) If $M$ is two-dimensional, then $\exp _{\text {id }}$ is actually defined on all of $T_{\mathrm{id}} \mathcal{D}_{\mu}^{s}(M)$ (a corollary of a famous result due originally to Wolibner [?]). If $M$ is three-dimensional, it is still notoriously unknown whether $\exp _{\text {id }}$ can be defined on the entire tangent space.

By right-invariance, the geodesic equation may be decomposed into two equations: the flow equation and the Euler equation. The flow equation relates material variables to spatial variables, and is given by

$$
\frac{\partial \eta}{\partial t}(t, x)=u(t, \eta(t, x))
$$

The Euler equation for the velocity field $u$ is

$$
\frac{\partial u}{\partial t}+P\left(\nabla_{u} u\right)=0
$$

where $P$ is the $L^{2}$ orthogonal projection of an arbitrary vector field onto the space of divergence-free vector fields, given explicitly by

$$
P(w)=w-\nabla \Delta^{-1} \operatorname{div} w .
$$

In three dimensions, it is often convenient to rewrite equation (2.5) (by taking the curl of both sides) as

$$
\frac{\partial}{\partial t} \operatorname{curl} u+[u, \operatorname{curl} u]=0 .
$$

The following was proved by the author in [?]. The main point is that the linearized Euler equation can be written in two ways, depending on whether one is righttranslating or left-translating the perturbed tangent vector to the identity. For different purposes, either equation may be preferred.

Proposition 2.1. The linearization of equation (2.5), obtained from a family $u(t, \sigma)$ of solutions by setting $z(t)=\left.\partial_{\sigma}\right|_{\sigma=0} u(t, \sigma)$, is

$$
\frac{\partial z}{\partial t}+P\left(\nabla_{z} u+\nabla_{u} z\right)=0
$$

with $P$ given by (2.6) to keep $z$ divergence-free. Writing $z=\eta_{*} v$, we obtain the equation

$$
P\left(\frac{\partial}{\partial t}(\Lambda v)+\omega_{o} \times v\right)=0,
$$

again with the consequence that $v$ is divergence-free. Here $\Lambda(t, x)=D \eta(t, x)^{T} D \eta(t, x)$ is the metric pullback and $\omega_{o}(x)=\operatorname{curl} u(0, x)$ is the initial vorticity.

In the study of fluid stability, the linearized Euler equation (2.8) is usually used alone to study Eulerian stability; however we can also study Lagrangian stability by incorporating the linearization of the flow equation (2.4). From the geometric perspective, this corresponds to studying the Jacobi equation along the geodesic. The fact that we 
can decouple the Jacobi equation this way was originally noticed by Rouchon [?]. It has been used to derive many properties of Jacobi fields and conjugate points; see for example [?].

Proposition 2.2. If $\eta(t)=\exp _{i d}\left(t u_{o}\right)$ is a geodesic in $\mathcal{D}_{\mu}(M)$ with velocity field $u(t)=$ $\frac{d \eta}{d t} \circ \eta(t)^{-1}$, then the Jacobi equation along $\eta$, right-translated to the identity, may be written as the pair of equations (2.8) and the linearized flow equation

$$
\frac{\partial y}{\partial t}+\nabla_{u} y-\nabla_{y} u=z .
$$

Here $\operatorname{div} y=0$ follows from the fact that $\operatorname{div} z=0$.

The differential of the exponential map may be written as

$$
d\left(\exp _{i d}\right)_{t u_{o}}\left(t z_{o}\right)=y(t) \circ \eta(t)
$$

where $y(t)$ is the solution of equations (2.8) and (2.10) with initial conditions $y(0)=0$ and $z(0)=z_{0}$.

Corollary 2.3. We may also write the differential of the exponential map as

$$
d\left(\exp _{i d}\right)_{t u_{o}}\left(v_{o}\right)=D \eta(t) w(t)
$$

where $w(t)$ solves the differential equation

$$
P\left(\frac{\partial}{\partial t}\left(\Lambda(t, x) \frac{\partial w}{\partial t}\right)+\omega_{o}(x) \times \frac{\partial w}{\partial t}\right)=0,
$$

with initial conditions $w(0)=0$ and $w^{\prime}(0)=v_{o}$.

Proof. We have $y(t)=\eta(t)_{*} w(t)$, and the equation $\frac{\partial y}{\partial t}+[u, y]=z$ is equivalent (by the definition of the Lie derivative) to $\frac{\partial}{\partial t}\left(\eta(t)_{*}^{-1} y(t)\right)=\eta(t)_{*}^{-1} z(t)$. Thus we have $\frac{\partial w}{\partial t}=v$, where $v$ satisfies $(2.9)$. The corollary follows.

We know that the exponential map is defined and $C^{\infty}$ on some neighborhood of the identity in $\mathcal{D}_{\mu}^{s}(M)$ for $s>n / 2+1$. It is not defined in the weak $L^{2}$ topology, since we do not have an existence and uniqueness theorem for solutions of the Euler equation with $L^{2}$ initial velocity. Thus the differential of the exponential map will only be defined only on some $\Omega \subset T_{\mathrm{id}} \mathcal{D}_{\mu}^{s}(M) \rightarrow T_{\exp \left(t u_{o}\right)} \mathcal{D}_{\mu}^{s}(M)$. However, if $u_{o}$ is sufficiently smooth, we can uniquely extend the differential to a continuous map on $T_{\mathrm{id}} \mathcal{D}_{\mu}^{0}(M)$ in the $L^{2}$ topology. (We emphasize that despite the notation, $\mathcal{D}_{\mu}^{0}(M)$ is not a topological group or a smooth manifold in any known sense.)

Definition 2.4. Let us denote by $T_{\eta} \mathcal{D}_{\mu}^{0}(M)$ the closure of $T_{\eta} \mathcal{D}_{\mu}(M)$ in the $L^{2}$ topology. For any fixed $a \in \mathbb{R}^{+}$and $u_{o} \in T_{\text {id }} \mathcal{D}_{\mu}(M)$, we set $E(a)=\left(d \exp _{\text {id }}\right)_{a u_{o}}$, and we define $\widetilde{E}(a): T_{\mathrm{id}} \mathcal{D}_{\mu}^{0}(M) \rightarrow T_{\exp \left(a u_{o}\right)} \mathcal{D}_{\mu}^{0}(M)$ to be the extension of $E(a)$ to the $L^{2}$-closure of $T_{\mathrm{id}} \mathcal{D}_{\mu}(M)$. (This is a closed subspace of the space of all $L^{2}$ vector fields, by [?].)

We can use $\widetilde{E}$ to extend the notion of conjugate points to the weak topology defined by the Riemannian metric. We say $\eta(a)$ is weakly monoconjugate to the identity if $\widetilde{E}(a)$ is not injective, and that $\eta(a)$ is weakly epiconjugate to the identity if $\widetilde{E}(a)$ is not 
surjective. Similarly, $\eta(a)$ is strongly monoconjugate if $E(a)$ is not injective, and $\eta(a)$ is strongly epiconjugate if $E(a)$ is not surjective.

The relations between weakly conjugate points are the same as those for conjugate points in strong metrics proved by Biliotti, Exel, Piccione, and Tausk [?], since those authors' proof relies only on the structure of the Jacobi equation rather than on global properties of infinite-dimensional manifolds. Thus we have:

Theorem 2.5 (Biliotti et al.). We have the following for weakly conjugate points:

- The set of weakly monoconjugate points is countable and dense in the set of weakly epiconjugate points;

- Every weakly monoconjugate point is also weakly epiconjugate;

- Any point that is strictly weakly epiconjugate (weakly epiconjugate and not weakly monoconjugate) is a limit point of weakly monoconjugate points.

These relations are not in general known to hold for strongly conjugate points in the present situation, where the Riemannian $L^{2}$ metric does not generate the $H^{s}$ topology in which we are working. However, we do have the following general results due to Grossmann [?]. These results rely only on the symmetry of the conjugacy relation.

Theorem 2.6 (Grossmann). Weakly and strongly conjugate points both satisfy the following relationships:

- Every (weakly/strongly) monoconjugate point is also a (weakly/strongly) epiconjugate point.

- If $\eta(a)$ is (weakly/strongly) epiconjugate to the identity, and if the range of $(\widetilde{E}(a), E(a))$ is closed, then $\eta(a)$ is also (weakly/strongly) monoconjugate to the identity.

In addition, the following relationships between weakly and strongly conjugate points are obvious: every strongly monoconjugate point is a weakly monoconjugate point (the kernel of the $H^{s}$ map is a subset of the kernel of the $L^{2}$ map), while every strongly epiconjugate point is a weakly epiconjugate point (the image of the $H^{s}$ map is a subset of the image of the $L^{2}$ map). The following proposition shows that for smooth geodesics in $\mathcal{D}_{\mu}(M)$, strictly weakly epiconjugate implies strongly epiconjugate. For the basic facts on Fredholm operators that we will use, we refer to Taylor's Appendix A [?].

Proposition 2.7. Suppose the initial velocity $u_{o} \in T_{i d} \mathcal{D}_{\mu}^{s}(M)$ is $C^{\infty}$, so that the geodesic $\eta(t)=\exp _{i d}\left(t u_{o}\right)$ is $C^{\infty}$ as long as it exists. If $\eta(a)$ is strictly weakly epiconjugate to id, then $\eta(a)$ is strongly epiconjugate to the identity.

Proof. Assume, to get a contradiction, that $\eta(a)$ is strictly weakly epiconjugate but not strongly epiconjugate to id.

We use the notation of Definition 2.4. By Proposition 2.6, $\eta(a)$ is strictly weakly epiconjugate if and only if the range of $\widetilde{E}(a)$ is not closed, which implies $\widetilde{E}(a)$ is not Fredholm. In addition, since $\eta(a)$ is not strongly epiconjugate to id, we know $E(a)$ is surjective, which means it must also be injective; therefore $E(a)$ is Fredholm. 
Thus if we left-translate the Jacobi operators, as

$$
\begin{aligned}
& F(a)=d L_{\eta(a)^{-1}} \circ E(a): T_{\mathrm{id}} \mathcal{D}_{\mu}^{s}(M) \rightarrow T_{\mathrm{id}} \mathcal{D}_{\mu}^{s}(M) \\
& \widetilde{F}(a)=d L_{\eta(a)^{-1}} \circ \widetilde{E}(a): T_{\mathrm{id}} \mathcal{D}_{\mu}^{0}(M) \rightarrow T_{\mathrm{id}} \mathcal{D}_{\mu}^{0}(M),
\end{aligned}
$$

then $F(a)$ is also Fredholm, while $\widetilde{F}(a)$ is not Fredholm. If we consider in addition the operator

$$
\bar{F}(a)=A^{s} F(a) A^{-s}: T_{\mathrm{id}} \mathcal{D}_{\mu}^{0}(M) \rightarrow T_{\mathrm{id}} \mathcal{D}_{\mu}^{0}(M),
$$

where $A=\left(1+\operatorname{curl}^{2}\right)^{1 / 2}$, then since $A^{s}$ is an isomorphism between $T_{\mathrm{id}} \mathcal{D}_{\mu}^{s}(M)$ and $T_{\text {id }} \mathcal{D}_{\mu}^{0}(M)$, we know $\bar{F}(a)$ is also Fredholm. The operators $\widetilde{F}(a)$ and $\bar{F}(a)$ are both defined in the same space, and thus we can consider their difference.

By Proposition 2.3, the operators $F(t)$ and $\widetilde{F}(t)$ both satisfy the differential equation

$$
P\left(\frac{d}{d t}\left(\Lambda(t) \frac{d F}{d t}\right)+\omega_{o} \times \frac{d F}{d t}\right)=0, \quad F(0)=0, \quad F^{\prime}(0)=\mathrm{id} .
$$

Thus the operator $\bar{F}(t)$ satisfies

$$
P\left(\frac{d}{d t}\left(\Lambda(t) A^{-s} \frac{d \bar{F}}{d t} A^{s}\right)+\omega_{o}\left(A^{-s} \frac{d \bar{F}}{d t} A^{s}\right)\right)=0
$$

where $A=\left(1+\operatorname{curl}^{2}\right)^{1 / 2}$. By considering commutators (and using the fact that $[A, P] \equiv$ $0)$, we have

$$
P\left[\frac{d}{d t}\left(\Lambda(t) \frac{d \bar{F}}{d t}\right)+\omega_{o}\left(\frac{d \bar{F}}{d t}\right)\right]=-P\left[\frac{d}{d t}\left(\left[A^{s}, \Lambda(t)\right] A^{-s} \frac{d \bar{F}}{d t}+\left[A^{s}, \omega_{o}\right] A^{-s} \bar{F}\right)\right]
$$

Now since $\Lambda(t)$ and $\omega_{o}$ are smooth operators (for any $t$ ), we know that $\left[A^{s}, \Lambda(t)\right]$ and $\left[A^{s}, \omega_{o}\right]$ are both differential operators of order less than $s$, by the product rule; hence $\left[A^{s}, \Lambda(t)\right] A^{-s}$ and $\left[A^{s}, \omega_{o}\right] A^{-s}$ are both compact operators on $L^{2}$. Now given that $\bar{F}$ is a continuous operator from $T_{\mathrm{id}} \mathcal{D}_{\mu}^{0} \subset L^{2}$ to itself, and given that $P$ is a smooth operator from $L^{2}$ to $T_{\text {id }} \mathcal{D}_{\mu}^{0} \subset L^{2}$, we can write

$$
P\left[\frac{d}{d t}\left(\Lambda(t)\left(\frac{d(\bar{F}-\tilde{F})}{d t}\right)\right)+\omega_{o}\left(\frac{d(\bar{F}-\tilde{F})}{d t}\right)\right]=K(t)
$$

where $K(t)$ is a fixed compact operator from $T_{\text {id }} \mathcal{D}_{\mu}^{0} \subset L^{2}$ to itself. Now $\widetilde{F}(0)=0$ and $\bar{F}(0)=0$; in addition $\widetilde{F}^{\prime}(0)=$ id and $\bar{F}^{\prime}(0)=\mathrm{id}$, so that $\widetilde{F}-\bar{F}$ vanishes to both first and second order.

Now consider the equation

$$
P\left[\frac{d}{d t}(\Lambda(t) q(t))+\omega_{o} q(t)\right]=0 .
$$

Since the operators are all bounded in $L^{2}$, this is a linear differential equation in $T_{\text {id }} \mathcal{D}_{\mu}^{0}(M)$, with solutions existing for all time; hence it has a bounded solution operator 
$G(t, \tau): T_{\mathrm{id}} \mathcal{D}_{\mu}^{0}(M) \rightarrow T_{\mathrm{id}} \mathcal{D}_{\mu}^{0}(M)$ such that $q(t)=G(t, \tau) q(\tau)$ for all $t, \tau \in \mathbb{R}$. By Duhamel's principle, we can then write

$$
\widetilde{F}(t)-\bar{F}(t)=\int_{0}^{t} \int_{0}^{\tau} G(\tau, \sigma) \circ K(\sigma) d \sigma d \tau .
$$

Since $K(\sigma)$ is compact for all $\sigma$ and $G(\tau, \sigma)$ is continuous, the composition is compact. The integrals are limits of sums of compact operators, hence also compact.

Now by assumption we know that $\bar{F}(t)$ is Fredholm while $\widetilde{F}(t)$ is not Fredholm; this is a contradiction since the sum of a Fredholm operator and a compact operator must be Fredholm.

We could also try to relate weak monoconjugacy to strong monoconjugacy, and this could be done most simply if we knew that every $L^{2}$ solution to (2.11) with $w(0)=0$ and $w(T)=0$ were actually $C^{\infty}$ as long as the flow and velocity field were $C^{\infty}$. (This would imply that any $L^{2}$ monoconjugate point corresponds to a zero of a $C^{\infty}$ Jacobi field, and in particular to a zero of an $H^{s}$ Jacobi field.) While this is true in many cases, it is not always true, due to the fact that the order of a monoconjugate point may be infinite.

The example of the 3-sphere from [?] is illustrative. In this example, we take a velocity field that is left-invariant under the standard group action on $S^{3}$; then it is automatically a steady solution of the Euler equation and so generates a geodesic of $\mathcal{D}_{\mu}\left(S^{3}\right)$. Monoconjugate points occur at $t=\frac{m \pi}{n}$, where $m$ and $n$ are any positive integers with $m \geq n$. Every such point has infinite order, and the infinite-dimensional space of Jacobi fields vanishing at both times is spanned by curl eigenfields, which are $C^{\infty}$. However, it is easy to find an infinite sum of such fields which converges in $L^{2}$ but not in $H^{s}$ for any $s>0$, just by choosing the coefficients correctly. Hence there is a Jacobi field vanishing at $t=0$ and $t=\frac{m \pi}{n}$ which is in $L^{2}$ but not in $H^{s}$ for any $s>0$.

In spite of this, it is still true that every weak monoconjugate point along this particular geodesic in $\mathcal{D}_{\mu}\left(S^{3}\right)$ is actually a strong monoconjugate point, and we believe it is likely that this is always true. However the example above shows the proof may be somewhat subtle.

The following theorem from [?] tells us when the first weakly conjugate point occurs, in terms of an ordinary differential equation.

Theorem 2.8. Suppose $\eta:[0, T) \rightarrow \mathcal{D}_{\mu}(M)$ is a geodesic. Let $u$ be the velocity field defined by $\frac{\partial \eta}{\partial t}(t, x)=u(t, \eta(t, x))$. Let us define $\Lambda(t, x)=D \eta(t, x)^{T} D \eta(t, x)$ and $\omega_{o}(x)=\operatorname{curl} u_{o}(x)$.

For each $x \in M$, let $\tau(x)>0$ be the first time such that

$$
\frac{d}{d t}\left(\Lambda(t, x) \frac{d u}{d t}\right)+\omega_{o}(x) \times \frac{d u}{d t}=0
$$

has a solution vanishing at $t=0$ and $t=\tau(x)$. Then the first weakly conjugate point to the identity along $\eta$ occurs at $\inf _{x \in M} \tau(x)$. This point is either strictly weakly 
epiconjugate (i.e., weakly epiconjugate but not weakly monoconjugate), or it is weakly monoconjugate of infinite order.

\section{Locating EPiconjugate Points}

Since the Jacobi equation is closely related to the linearized Euler equation by Proposition 2.2, we can use techniques developed by Lifschitz and Hameiri [?] and Friedlander and Vishik [?] for the latter. These authors showed that solutions of the linearized Euler equation for sharply peaked initial data could be approximated near the peak by a certain ordinary differential equation, using a WKB approximation. Lifschitz-Hameiri proved a weaker estimate that is valid more generally than that of Friedlander-Vishik, but one that is sufficient for our purposes. (Friedlander-Vishik's estimate is valid only for steady solutions of the Euler equation on the flat torus $\mathbb{T}^{3}$, while Lifschitz-Hameiri is valid for all solutions in any geometry.)

The basic technique of WKB analysis is to posit a solution in the form

$$
z=e^{i \Phi / \varepsilon} a+r
$$

with $\varepsilon$ a small parameter, and expand in powers of $\varepsilon$ to obtain simple equations for $\Phi$ and $a$. Then we prove that the error term $r$ is bounded by $\varepsilon$. The following computation and error estimate was performed by Lifschitz and Hameiri [?].

Theorem 3.1 (Lifschitz-Hameiri). Suppose $u$ is a (possibly time-dependent) solution of the Euler equation (2.5) on a compact manifold $M$, on some time interval $[0, T]$.

If $z=e^{i \Phi / \varepsilon} a+r$ solves the linearized Euler equation

$$
\frac{\partial z}{\partial t}+\nabla_{u} z+\nabla_{z} u=2 \nabla \Delta^{-1} \operatorname{div}\left(\nabla_{z} u\right), \quad \operatorname{div} z=0
$$

with initial condition $z(0)=e^{i \Phi_{o} / \varepsilon} a_{o}$, then for $\varepsilon$ small, the dominant terms satisfy

$$
\frac{\partial \Phi}{\partial t}+u(\Phi)=0
$$

and

$$
\frac{\partial a}{\partial t}+\nabla_{u} a+\nabla_{a} u=2 q \nabla \Phi
$$

where

$$
q=\frac{\left\langle\nabla_{a} u, \nabla \Phi\right\rangle}{\langle\nabla \Phi, \nabla \Phi\rangle}
$$

while the remainder term $r$ satisfies

$$
\|r(t)\|_{L^{2}} \leq C \varepsilon
$$

for all $t \in[0, T]$, where $C$ is a constant that depends on $T$ and derivatives of $\Phi$ and a. 
Equations (3.17) and (3.18) look like partial differential equations, but they are more properly thought of as ordinary differential equations, which makes analyzing their solutions simpler. We can write them using right-translation as ODEs along a particular Lagrangian path, or alternatively using left-translation as ODEs in a particular tangent space.

Proposition 3.2. If $u$ is a solution of the Euler equation (2.5) with flow $\eta$, then we can write the solution of equation (3.17) as $\Phi(t)=\Phi_{o} \circ \eta(t)^{-1}$ with $\nabla \Phi(t)=\left(D \eta(t)^{-1}\right)^{\star} \nabla \Phi_{o}$. In addition, we have for each $x$ the formula $a(t, \eta(t, x))=\alpha(t)$ where $\alpha(t)$ is a vector field along $t \mapsto \eta(t, x)$ satisfying the equation

$$
\frac{D \alpha}{d t}+\nabla_{\alpha(t)} u(t)=2 \frac{\left\langle\nabla_{\alpha(t)} u(t), \nabla \Phi(t)\right\rangle}{\langle\nabla \Phi(t), \nabla \Phi(t)\rangle} \nabla \Phi(t), \quad \alpha(0)=a(0, x), \quad\langle\alpha(t), \nabla \Phi(t)\rangle \equiv 0 .
$$

Alternatively, for each $x_{o}$ we can write $a(t, \eta(t, x))=D \eta(t, x)(\beta(t))$, where $\beta(t) \in$ $T_{x_{o}} M$ for all $t$ and $\beta(t)$ satisfies

$$
\frac{d}{d t}\left(\pi_{\xi_{o}^{\perp}} \Lambda(t) \beta(t)\right)+\left\langle\xi_{o}, \omega_{o}\right\rangle \xi_{o} \times \beta(t)=0, \quad \beta(0)=a(0, x), \quad\left\langle\beta(t), \xi_{o}\right\rangle \equiv 0,
$$

where $\xi_{o}=\nabla \Phi_{o}(x)$ and $\omega_{o}=\operatorname{curl} u_{o}(x)$.

Proof. The equation $\frac{\partial \Phi}{\partial t}+u(\Phi)=0$ can be rewritten as $\frac{\partial}{\partial t}(\Phi(t) \circ \eta(t))=0$, which implies the solution $\Phi(t)=\Phi_{o} \circ \eta(t)^{-1}$. The formula for $\nabla \Phi(t)$ is a consequence of the chain rule. Finally the formula

$$
\frac{D}{d t} a(t, \eta(t, x))=\frac{\partial a}{\partial t}(t, \eta(t, x))+\nabla_{u(t, \eta(t, x))} a(t, \eta(t, x))
$$

allows us to write $(3.18)$ as the ordinary differential equation $(3.21)$.

To obtain (3.22) from (3.21), we apply $(D \eta)^{\mathrm{T}}$ to both sides of (3.21) and obtain

$$
(D \eta)^{\mathrm{T}}\left(\frac{D \alpha}{d t}+\nabla_{\alpha} u\right)=c \xi_{o}
$$

for some function $c$. The left side was computed in [?] to be, with $\alpha=D \eta(\beta)$,

$$
(D \eta)^{\mathrm{T}}\left(\frac{D \alpha}{d t}+\nabla_{\alpha} u\right)=\frac{d}{d t}(\Lambda(t) \beta(t))+\omega_{o} \times \beta(t),
$$

using conservation of vorticity. Applying $\pi_{\xi_{o}^{\perp}}$ to both sides of 3.23 gives 3.22 .

Corollary 3.3. If $w(t)$ is a solution of equation (2.11) with initial condition $w(0, x) \equiv 0$ and $w_{t}(0, x)=e^{i \Phi_{o}(x) / \varepsilon} v_{o}(x)$, then $w(t, x)=e^{i \Phi_{o}(x) / \varepsilon} \int_{0}^{t} \beta(\tau, x) d \tau+\tilde{r}(t, x)$, where $\beta(t, x)$ satisfies 3.22 with initial condition $\beta(0, x)=v_{o}(x)$ and $\|\tilde{r}(t)\|_{L^{2}} \leq \tilde{C} \varepsilon$ for $t \in[0, T]$. Here $\tilde{C}$ a constant depending on $T$ and derivatives of $\eta$ and $v_{o}$.

Proof. We have $w(t, x)=\int_{0}^{t} v(\tau, x) d \tau$ and

$$
v(t)=\eta(t)_{*}^{-1} z(t)=e^{i \Phi_{o} / \varepsilon} \eta(t)_{*}^{-1} a(t)+\eta(t)_{*}^{-1} r(t),
$$


where $a$ satisfies (3.18). Thus

$$
\begin{aligned}
\left\|w(t)-e^{i \Phi_{o} / \varepsilon} \beta(t)\right\|_{L^{2}} & \leq \int_{0}^{t}\left\|\eta(\tau)_{*}^{-1} r(\tau)\right\| d \tau \\
& \leq C \varepsilon \int_{0}^{t}\left\|\eta(\tau)_{*}^{-1}\right\|_{L^{2}} d \tau \\
& \leq C \varepsilon \int_{0}^{t}\left\|\eta(\tau)^{-1}\right\|_{C^{1}} d \tau
\end{aligned}
$$

since $\eta$ is volume-preserving. With $\tilde{C}=C \int_{0}^{t}\left\|\eta(\tau)^{-1}\right\|_{C^{1}} d \tau$, we are done.

The main theorem of the paper follows.

Theorem 3.4. Let $\eta$ be a smooth geodesic in $\mathcal{D}_{\mu}(M)$ with Eulerian velocity field $u$. If for some $x_{o} \in M$ and some unit-length $\xi_{o} \in T_{x_{o}} M$, the equation

$$
\frac{d}{d t}\left(\pi_{\xi_{o}^{\perp}} \Lambda\left(t, x_{o}\right) \frac{d \gamma}{d t}\right)+\left\langle\omega_{o}\left(x_{o}\right), \xi_{o}\right\rangle \xi_{o} \times \frac{d \gamma}{d t}=0, \quad\left\langle\gamma(t), \xi_{o}\right\rangle \equiv 0
$$

has a solution with $\gamma(0)=0$ and $\gamma(a)=0$, then $\eta(a)$ is weakly epiconjugate to the identity along $\eta$.

Proof. Let $\delta>0$ be any number small enough that Riemannian normal coordinates exist in a $\delta$-neighborhood of $x_{o}$. Choose normal coordinates $\left(x_{1}, x_{2}, x_{3}\right)$ with axes aligned so that $\xi_{o}=\left.\partial_{x_{1}}\right|_{x_{o}}$ and $\gamma^{\prime}(0)=\left.\partial_{x_{3}}\right|_{x_{o}}$, where $\gamma$ is the solution of 3.24 with $\gamma(0)=\gamma(a)=0$. Define $\Phi_{o}: B_{\delta}\left(x_{o}\right) \rightarrow \mathbb{R}$ by $\Phi_{o}\left(x_{1}, x_{2}, x_{3}\right)=x_{1}$, and set $\xi_{o} \equiv \nabla \Phi_{o}=$ $\partial_{x_{1}}+O\left(\delta^{2}\right)$. (We could extend $\Phi_{o}$ globally with a bump function, but this is not necessary, since we will only be working in this neighborhood.) Let $\psi: \mathbb{R} \rightarrow \mathbb{R}$ be a bump function with $\psi \equiv 1$ in $\left[-\frac{1}{2}, \frac{1}{2}\right], \psi \equiv 0$ on $\mathbb{R} \backslash(-1,1)$, and $\left|\psi^{\prime}\right| \leq 3$ everywhere. Define a function $\alpha: M \rightarrow \mathbb{R}$ by $\alpha\left(x_{1}, x_{2}, x_{3}\right)=x_{2} \psi\left(\frac{x_{1}^{2}+x_{3}^{2}}{\delta^{2}}+\frac{x_{2}^{2}}{\delta^{4}}\right)$ (and zero outside the coordinate neighborhood). Finally, define $v_{o}=\xi \times \nabla \alpha$. Then in the support of $v_{o}$, we must have $x_{1}<\delta, x_{2}<\delta^{2}$, and $x_{3}<\delta$. As a result, we have

$$
\begin{aligned}
\nabla \alpha=\frac{2 x_{1} x_{2}}{\delta^{2}} \psi^{\prime}\left(\frac{x_{1}^{2}+x_{3}^{2}}{\delta^{2}}+\frac{x_{2}^{2}}{\delta^{4}}\right) \partial_{x_{1}}+\frac{2 x_{3} x_{2}}{\delta^{2}} \psi^{\prime}\left(\frac{x_{1}^{2}+x_{3}^{2}}{\delta^{2}}+\frac{x_{2}^{2}}{\delta^{4}}\right) \partial_{x_{3}} \\
+\left[\psi\left(\frac{x_{1}^{2}+x_{3}^{2}}{\delta^{2}}+\frac{x_{2}^{2}}{\delta^{4}}\right)+\frac{2 x_{2}^{2}}{\delta^{4}} \psi^{\prime}\left(\frac{x_{1}^{2}+x_{3}^{2}}{\delta^{2}}+\frac{x_{2}^{2}}{\delta^{4}}\right)\right] \partial_{x_{2}}+O\left(\delta^{2}\right),
\end{aligned}
$$

and since $x_{1} x_{2}=O\left(\delta^{3}\right)$ and $x_{3} x_{2}=O\left(\delta^{3}\right)$, we have

$$
\nabla \alpha=\left[\psi\left(\frac{x_{1}^{2}+x_{3}^{2}}{\delta^{2}}+\frac{x_{2}^{2}}{\delta^{4}}\right)+\frac{2 x_{2}^{2}}{\delta^{4}} \psi^{\prime}\left(\frac{x_{1}^{2}+x_{3}^{2}}{\delta^{2}}+\frac{x_{2}^{2}}{\delta^{4}}\right)\right] \partial_{x_{2}}+O(\delta) .
$$

Therefore,

$$
v_{o}\left(x_{1}, x_{2}, x_{3}\right)=\left[2 \frac{x_{2}^{2}}{\delta^{4}} \psi^{\prime}\left(\frac{x_{1}^{2}+x_{3}^{2}}{\delta^{2}}+\frac{x_{2}^{2}}{\delta^{4}}\right)+\psi\left(\frac{x_{1}^{2}+x_{3}^{2}}{\delta^{2}}+\frac{x_{2}^{2}}{\delta^{4}}\right)\right] \partial_{x_{3}}+O(\delta) .
$$


Since $x_{2}=O\left(\delta^{2}\right), \psi=O(1)$, and $\psi^{\prime}=O(1)$, both terms in $v_{o}$ are $O(1)$ in the support of $v_{o}$.

Let us write $\Gamma(t, x): \xi_{o}(x)^{\perp} \subset T_{x} M \rightarrow \xi_{o}(x)^{\perp}$ for the solution operator of

$$
\frac{\partial}{\partial t}\left(\pi_{\xi_{o}(x)^{\perp}} \Lambda(t, x) \frac{\partial \gamma}{\partial t}(t, x)\right)+\frac{\left\langle\omega_{o}(x), \xi_{o}(x)\right\rangle}{\left\langle\xi_{o}(x), \xi_{o}(x)\right\rangle} \xi_{o}(x) \times \frac{\partial \gamma}{\partial t}(t, x)=0
$$

with initial conditions $\Gamma(0, x)=0$ and $\partial_{t} \Gamma(0, x)=\mathrm{id}$, i.e., $\Gamma(t, x)\left(\chi_{o}\right)=\gamma(t)$ where $\gamma(0)=0$ and $\dot{\gamma}(0)=\chi_{o}$. Then $\Gamma(t, x)$ is smooth and does not depend on $\delta$; thus we can write $\Gamma(a, x)=\Gamma\left(a, x_{o}\right)+O(\delta)$. By assumption and our coordinate construction, we have $\Gamma\left(a, x_{o}\right)\left(\partial_{z}\right)=0$. Since $\Gamma\left(t, x_{o}\right)$ is an operator only in the space perpendicular to $\xi_{o}=\left.\partial_{x_{1}}\right|_{x_{o}}$, and since $\Gamma\left(a, x_{o}\right)\left(\partial_{x_{3}}\right)=0$ by construction of our coordinates, we have for any $\chi \in \xi_{o}\left(x_{o}\right)^{\perp}$ that $\Gamma\left(a, x_{o}\right)(\chi)=\left\langle\chi, \partial_{x_{2}}\right\rangle \Gamma\left(a, x_{0}\right)\left(\partial_{x_{2}}\right)+O(\delta)$.

In particular for the $v_{o}$ constructed above, we have

$$
\Gamma(a, x)\left(v_{o}(x)\right)=\left\langle v_{o}(x), \partial_{x_{2}}\right\rangle \Gamma\left(a, x_{o}\right)\left(\partial_{x_{2}}\right)+O(\delta)=O(\delta),
$$

so that

$$
\left\|\Gamma(a, x)\left(v_{o}(x)\right)\right\|_{L^{2}}=O(\delta) \cdot \operatorname{vol}\left[\operatorname{supp}\left(v_{o}\right)\right]=O\left(\delta^{5}\right) .
$$

On the other hand, we have $\left\|v_{o}\right\|_{L^{2}}=O\left(\delta^{4}\right)$ since $v_{o}=O(1)$ on $\operatorname{supp}\left(v_{o}\right)$.

By Corollary 3.3, we have

$$
\|w(a)\|_{L^{2}} \leq B \delta^{5}+\tilde{C} \varepsilon
$$

for some constant $B$. Since $\tilde{C}$ depends on derivatives of $v_{o}$, it may have a $\delta$-dependence; however we are still free to choose $\varepsilon$, and thus we can choose it small enough so that $\|w(a)\|_{L^{2}}=O\left(\delta^{5}\right)$. On the other hand, we still have $\left\|w^{\prime}(0)\right\|_{L^{2}}=O\left(\delta^{4}\right)$.

Now we consider a normalized sequence $w_{n}$ of solutions with $\delta_{n}=\frac{1}{n^{2}}$ and $\varepsilon_{n}$ chosen so that $\left\|w_{n}(a)\right\|_{L^{2}}=O\left(\frac{1}{n^{2}}\right)$ and $\left\|w_{n}^{\prime}(0)\right\|_{L^{2}}=1$. Then the series $\sum_{n=1}^{\infty} w_{n}(a)$ converges to some divergence-free vector field in $L^{2}$. We now have two possibilities: either $\widetilde{E}(a)$ is injective, or it is not. If $\widetilde{E}(a)$ is not injective, then $\eta(a)$ is weakly monoconjugate to id and thus also weakly epiconjugate to id, and we are done. Otherwise, if $\widetilde{E}(a)$ is injective, then we have $\sum_{n=1}^{\infty} \widetilde{E}(a)\left(w_{n}^{\prime}(0)\right)$ convergent in $L^{2}$, while $\sum_{n=1}^{\infty} w_{n}^{\prime}(0)$ cannot converge in $L^{2}$, so that $\widetilde{E}(a)$ does not have closed range. Hence $\eta(a)$ must be weakly epiconjugate to id. Thus we are done.

Remark 3.5. In the proof of Theorem 3.4, we assumed for simplicity that the dimension of $M$ is 3. However the theorem holds generally as long as $\operatorname{dim} M \geq 2$. In the more general case we need to replace the operator $\chi \mapsto \operatorname{curl} u_{o} \times \chi$ with $\chi \mapsto\left(\iota_{\chi} d u_{o}^{b}\right)^{\sharp}$, where the generalized vorticity cross product is defined in arbitrary dimensions to satisfy (for any $\zeta$ )

$$
\left\langle\left(\iota_{\chi} d u_{o}^{b}\right)^{\sharp}, \zeta\right\rangle=d X_{o}^{b}(\chi, \zeta)=\left\langle\nabla_{\chi} u_{o}, \zeta\right\rangle-\left\langle\nabla_{\zeta} u_{o}, \chi\right\rangle .
$$

With this modification, all other parts of the proof are valid, with the expression $x_{1}^{2}+x_{3}^{2}$ replaced whenever it appears with $x_{1}^{2}$ (in dimension two) or $x_{1}^{2}+x_{3}^{2}+x_{4}^{2}+\cdots+x_{n}^{2}$ (in dimension $n \geq 3$ ). 
However, in dimension two the theorem happens not to give any useful information. This is because we must have $\left\langle\gamma, \xi_{o}\right\rangle \equiv 0$, which in two dimensions implies that $\gamma=$ $f \xi_{o}^{\perp}$ for some function $f$. (Here $\xi_{o}^{\perp}$ is the standard rotation by $90^{\circ}$.) As a result, $d X_{o}^{\mathrm{b}}(\gamma, \dot{\gamma})=f \dot{f} d X_{o}^{\mathrm{b}}\left(\xi_{o}^{\perp}, \xi_{o}^{\perp}\right)=0$ regardless of the function $f$, so that equation (3.24) becomes

$$
\frac{d}{d t}\left(\left\langle\xi_{o}^{\perp}, \Lambda\left(t, x_{o}\right) \xi_{o}^{\perp}\right\rangle \frac{d f}{d t}\right)=0 .
$$

This equation clearly has no solutions $f(t)$ vanishing at two distinct times, due to positive-definiteness of $\Lambda$, and hence Theorem 3.4 does not yield any epiconjugate points at all in dimension two.

This is as expected, since the exponential map is Fredholm in dimension two [?]. If there were nontrivial epiconjugate points obtainable by Theorem 3.4, it would imply (as shown in the next section) that epiconjugate points could occur in intervals along a geodesic; but Fredholmness of the exponential map implies that conjugate points must be isolated along a geodesic.

On the other hand, the fact that the same technique works and yields genuine epiconjugate points in any dimension higher than three implies that Fredholmness must also fail in any dimension higher than three.

Although Theorem 3.4 allows us to locate many of the conjugate points along a particular geodesic, it will typically not get all of them. Essentially, the reason for this is the following: the first conjugate point is obtained from the solution of a threedimensional second-order differential equation at some point, by Theorem 2.8, while the epiconjugate points detectable by the WKB method all come from the solution of a two-dimensional second-order differential equation. In essence, we are looking at the vanishing of the index form

$$
I_{0, \tau}(\gamma, \gamma)=\int_{0}^{\tau}\left\langle\Lambda(t, x) \frac{d \gamma}{d t}, \frac{d \gamma}{d t}\right\rangle+\left\langle\omega_{o}(x) \times \gamma(t), \frac{d \gamma}{d t}\right\rangle d t
$$

among vectors $\gamma(t)$ vanishing at $t=0$ and $t=\tau$. Both problems have the same index form, but the WKB problem forces the vectors $\gamma(t)$ to all be orthogonal to the same fixed vector. Thus there are fewer solutions.

Explicitly, suppose the first conjugate point location $\tau$ along a geodesic is found from equation (2.15), such that that equation has only one solution vanishing at both $t=0$ and $t=\tau$, with nontrivial variation in all three directions. Then no two-dimensional vector solution can make the index form vanish, and thus the first epiconjugate point coming from Theorem 3.4 must be beyond the first conjugate point coming from Theorem 2.8. We construct such an example in the following.

Example 3.6. Consider the annular solid torus $S^{1} \times S^{1} \times[a, b]$ (the region enclosed by two concentric tori) with coordinate system $(x, y, z)$ in which the metric is given by

$$
d s^{2}=z d x^{2}+(d y+f(x) d z)^{2}+d z^{2},
$$


for some periodic function $f(x)$. If $u=\partial_{x}$ in this coordinate system, then $\operatorname{curl} u=\partial_{y}$, so that we automatically have $[u, \operatorname{curl} u]=0$, which implies by (2.7) that $u$ is a steady solution of the Euler equation.

In the orthonormal basis $e_{1}=z^{-1 / 2} \partial_{x}, e_{2}=\partial_{y}, e_{3}=z^{1 / 2}\left(\partial_{z}-f(x) \partial_{y}\right)$, the operators in (2.15) take the form

$$
\Lambda(t, x, y, z)=\left(\begin{array}{ccc}
1 & 0 & 0 \\
0 & 1 & k(t, x, z) \\
0 & k(t, x, z) & 1+k(t, x, z)^{2}
\end{array}\right) \quad \text { and } \quad \omega_{o}(x, y, z)=\left(\begin{array}{ccc}
0 & 0 & 1 \\
0 & 0 & 0 \\
-1 & 0 & 0
\end{array}\right) \text {, }
$$

where $k(t, x, z)=\sqrt{z}(f(t+x)-f(x))$. The solution operator $\Upsilon$ of (2.15) satisfying $\Upsilon(0)=0$ and $\Upsilon^{\prime}(0)=$ id is

$$
\Upsilon(t)=\left(\begin{array}{ccc}
\sin t & F(t) \sin t-G(t) \cos t & \cos t-1 \\
-G(t) & t+\int_{0}^{t} k(\tau)^{2} d \tau+\int_{0}^{t}\left[G(\tau) F^{\prime}(\tau)-F(\tau) G^{\prime}(\tau)\right] d \tau & -F(t) \\
1-\cos t & -F(t) \cos t-G(t) \sin t & \sin t
\end{array}\right)
$$

where $F(t)=\int_{0}^{t} k(\tau) \cos \tau d \tau$ and $G(t)=\int_{0}^{t} k(\tau) \sin \tau d \tau$ (suppressing the dependence on $x$ and $z$ for simplicity).

There is a conjugate point at $t$ iff there is some vector $v_{o}$ with $\Upsilon(t)\left(v_{o}\right)=0$, i.e., iff $\operatorname{det} \Upsilon(t)=0$. We can easily compute

$$
\begin{aligned}
\operatorname{det} \Upsilon(t)=- & \sin t\left[F(t)^{2}+G(t)^{2}\right] \\
+ & 2(1-\cos t)\left[t+\int_{0}^{t} k(\tau)^{2} d \tau+\int_{0}^{t}\left[G(\tau) F^{\prime}(\tau)-F(\tau) G^{\prime}(\tau)\right] d \tau\right] .
\end{aligned}
$$

For a particular example, e.g., $f(x)=\sin x$, it is easy to check numerically that for any $(x, y, z)$, the first time where $\operatorname{det} \Upsilon(t)=0$ is $t=2 \pi$. (This is probably true in general, but it is not important here).

The unique (up to rescaling) vector $v_{o}$ for which $\Upsilon(2 \pi)\left(v_{o}\right)=0$ is

$$
v_{o}=\left(\begin{array}{c}
F(2 \pi) \\
0 \\
-G(2 \pi)
\end{array}\right)
$$

and the corresponding solution to $(2.15)$ is

$$
\gamma(t)=\left(\begin{array}{c}
F(2 \pi) \sin t+G(2 \pi)(1-\cos t) \\
G(2 \pi) F(t)-F(2 \pi) G(t) \\
F(2 \pi)(1-\cos t)-G(2 \pi) \sin t
\end{array}\right) .
$$

There is no vector $\xi_{o}$ for which $\left\langle\gamma(t), \xi_{o}\right\rangle=0$ for all $t$. As a result, the reduced index form does not vanish at $t=2 \pi$, and so the WKB method does not predict the epiconjugate point at $t=2 \pi$. 


\section{Conjugate Point intervals}

We now explore the consequences of Theorem 3.4. First, we have an easy theorem on continuity of conjugate point locations with respect to the fixed initial vector $\xi_{o}$.

Theorem 4.1. If for some $x_{o} \in M$ and some unit vector $\xi_{o} \in T_{x_{o}} M$, the equation (3.24) has a solution $\gamma(t)$ with $\gamma(0)=0$ and $\gamma\left(t_{o}\right)=0$, then for any $\tilde{\xi}_{o}$ sufficiently close to $\xi_{o}$, there is a solution $\tilde{\gamma}(t)$ with $\tilde{\gamma}(0)=0$ and $\tilde{\gamma}\left(\tilde{t}_{o}\right)=0$ for some $\tilde{t}_{o}$.

Proof. Fix an orthonormal basis of vector fields $e_{1}, e_{2}$, and $e_{3}$ in a neighborhood of $x_{o}$. Using spherical coordinates $\theta$ and $\phi$, we can write for any $x$ near $x_{o}$

$$
\begin{aligned}
& \xi_{o}=\left.\sin \theta \cos \phi e_{1}\right|_{x}+\left.\sin \theta \sin \phi e_{2}\right|_{x}+\left.\cos \theta e_{3}\right|_{x} \\
& \xi_{1}=\left.\cos \theta \cos \phi e_{1}\right|_{x}+\left.\cos \theta \sin \phi e_{2}\right|_{x}-\left.\sin \theta e_{3}\right|_{x} \\
& \xi_{2}=-\left.\sin \phi e_{1}\right|_{x}+\left.\cos \phi e_{2}\right|_{x} .
\end{aligned}
$$

Then any vector orthogonal to $\xi_{o}$ must be a linear combination of $\xi_{1}$ and $\xi_{2}$, so that the equation 3.24 becomes, with $u(t)=f(t) \xi_{1}+g(t) \xi_{2}$,

$$
\begin{aligned}
\frac{d}{d t}\left[\left(\begin{array}{cc}
\left\langle\xi_{1}, \Lambda(t, x) \xi_{1}\right\rangle & \left\langle\xi_{1}, \Lambda(t, x) \xi_{2}\right\rangle \\
\left\langle\xi_{1}, \Lambda(t, x) \xi_{2}\right\rangle & \left\langle\xi_{2}, \Lambda(t, x) \xi_{2}\right\rangle
\end{array}\right)\left(\begin{array}{c}
\dot{f}(t) \\
\dot{g}(t)
\end{array}\right)\right] \\
+\left\langle\omega_{o}(x) \times \xi_{1}, \xi_{2}\right\rangle\left(\begin{array}{cc}
0 & 1 \\
-1 & 0
\end{array}\right)\left(\begin{array}{c}
\dot{f}(t) \\
\dot{g}(t)
\end{array}\right)=\left(\begin{array}{l}
0 \\
0
\end{array}\right)
\end{aligned}
$$

In this way, we can consider the dependence of the conjugate time $t_{o}$ on $\theta, \phi$, and $x$.

By the general theory of oscillation for self-adjoint systems (see Reid [?]), equation 4.27) has a solution satisfying $u(0)=0$ and $u\left(t_{o}\right)=0$ if and only if the index form

$$
\begin{aligned}
& \text { (4.28) } \quad I_{T}\left(f \xi_{1}+g \xi_{2}, f \xi_{1}+g \xi_{2}\right)= \\
& \int_{0}^{T}\left(\tilde{\Lambda}_{11}(t, \theta, \phi) \dot{f}(t)^{2}+2 \tilde{\Lambda}_{12}(t, \theta, \phi) \dot{f}(t) \dot{g}(t)+\tilde{\Lambda}_{22}(t, \theta, \phi) \dot{g}(t)^{2}+2 \tilde{\omega}_{o}(\theta, \phi) f(t) \dot{g}(t)\right) d t
\end{aligned}
$$

defined for vector functions $u(t)$ vanishing at $t=0$ and $t=T$, is negative on some subspace for $T>t_{o}$ and positive-definite for $T<t_{o}$. (Here, for each $x \in M$, we have $\tilde{\Lambda}_{i j}(t, \theta, \phi)=\left\langle\xi_{i}, \Lambda(t) \xi_{j}\right\rangle$ and $\left.\tilde{\omega}_{o}(\theta, \phi)=\left\langle\omega_{o} \times \xi_{1}, \xi_{2}\right\rangle.\right)$

In fact, if we set

$$
J(\theta, \phi)(\gamma)=\frac{I_{T}(\gamma, \gamma)}{\int_{0}^{T}|\gamma(t)|^{2} d t},
$$

then for any fixed $\theta_{o}$ and $\phi_{o}$, we will have for any $T<t_{o}$ that the infimum satisfies $\inf _{\gamma} J\left(\theta_{o}, \phi_{o}\right)(\gamma)=\lambda\left(T, \theta_{o}, \phi_{o}\right)>0$, while if $T>t_{o}$, then $\inf _{\gamma} J\left(\theta_{o}, \phi_{o}\right)(\gamma)=$ $\lambda\left(T, \theta_{o}, \phi_{o}\right)<0$. We can prove this by noting that, by standard Sturm-Liouville theory for self-adjoint systems, $J(\theta, \phi)(\gamma)$ attains a minimum at a certain eigenvector field $\gamma(t)$ of a self-adjoint operator, and the minimum is an eigenvalue which we can denote by $\lambda(T, \theta, \phi)$. This eigenvalue depends continuously on $T, \theta$, and $\phi$ by the usual theory. 
As a result, for any fixed $T<t_{o}$, if $\theta$ and $\phi$ are sufficiently close to $\theta_{o}$ and $\phi_{o}$, we will have $\lambda(T, \theta, \phi)>0$; and for any fixed $T>t_{o}$ we will have $\lambda(T, \theta, \phi)<0$. As a result, by continuity with respect to $T$ we must have $\lambda\left(\tilde{t}_{o}, \theta, \phi\right)=0$ for some $\tilde{t}_{o}$.

The theorem above gives existence of (possibly multivalued) functions $t$ of spherical variables. For any fixed $\theta_{o}$ and $\phi_{o}$ we have an open domain in the sphere, containing $\theta_{o}$ and $\phi_{o}$, on which $t(\theta, \phi)$ is defined. Hence $t(\theta, \phi)$ is defined on some open subset of the 2-sphere. We can understand its behavior better by examining the differential equation directly, as follows. As a consequence, we will prove that $t$ is a differentiable function of $\theta$ and $\phi$.

Proposition 4.2. At a point $x_{o} \in M$ and for a unit $\xi_{o} \in T_{x_{o}} M$ with $\left\langle\xi_{o}, \omega_{o}\left(x_{o}\right)\right\rangle \neq 0$, the equation (3.24) has a solution with $\gamma(0)=0$ and $\gamma(a)=0$ if and only if the solution of the $2 \times 2$ matrix equation

$$
\frac{d W}{d t}+\left\langle\omega_{o}\left(x_{o}\right), \xi_{o}\right\rangle J\left(\pi_{\xi_{o}^{\perp}} \Lambda\left(t, x_{o}\right) \pi_{\xi_{o}^{\perp}}\right)^{-1} W=0
$$

with $W(0)=i d$ satisfies $\operatorname{Tr} W(a)=2$. Here $J=\left(\begin{array}{cc}0 & -1 \\ 1 & 0\end{array}\right)$.

Proof. Let us write the solution $\gamma(t)$ of 3.24 with $\gamma(0)=0$ and $\gamma^{\prime}(0)=v_{o}$ as a matrix operator $u(t)=S(t)\left(v_{o}\right)$, with $S(t): \xi_{o}^{\perp} \rightarrow \xi_{o}^{\perp}$. Then the $2 \times 2$ matrix $\Gamma$ must satisfy

$$
\frac{d}{d t}\left(\pi_{\xi_{o}^{\perp}} \Lambda \frac{d S}{d t}\right)+\left\langle\omega_{o}, \xi_{o}\right\rangle J \frac{d S}{d t}=0
$$

with $S(0)=0$ and $\frac{d S}{d t}(0)=\mathrm{id}$.

Writing $W=\pi_{\xi_{o}^{\perp}} \Lambda \frac{d S}{d t}$ and using the fact that $\Lambda(0)=\mathrm{id}$, we have the initial condition $W(0)=\mathrm{id}$, while $W$ satisfies 4.29 . In addition, since $\pi_{\xi_{o}}^{\perp} \Lambda \pi_{\xi_{o}}^{\perp}$ is symmetric and $J$ is antisymmetric, we have $\operatorname{Tr} \frac{d W}{d t} W^{-1}=0$, so that $\operatorname{det} W(t) \equiv 1$ for all $t$. On the other hand, if we integrate 4.30 in time, we obtain

$$
W(t)+\left\langle\xi_{o}, \omega_{o}\right\rangle J S(t)=\mathrm{id} .
$$

Since by assumption $\left\langle\xi_{o}, \omega_{o}\right\rangle \neq 0$, there is a $v_{o} \in \xi_{o}^{\perp}$ with $S(a)\left(v_{o}\right)=0$ if and only if $W(a)$ has 1 as an eigenvalue. Since $W$ is a $2 \times 2$ matrix with $\operatorname{det} W=1$, the eigenvalues of $W$ satisfy $\lambda^{2}-\operatorname{Tr} W(a) \lambda+1=0$, so that $\lambda=1$ is a solution if and only if $\operatorname{Tr} W(a)=2$.

Now to study differentiability, we fix the dependence of the solution on $\xi_{o} \in S^{2}$ as in Theorem 4.1; then equation (4.30) becomes

$$
\frac{\partial W(t, \theta, \phi)}{\partial t}+J \Theta(t, \theta, \phi) W(t, \theta, \phi)=0,
$$

with $\Theta(t, \theta, \phi)=\left\langle\omega_{o}\left(x_{o}\right), \xi_{o}\right\rangle\left(\pi_{\xi_{o}^{\perp}} \Lambda\left(t, x_{o}\right) \pi_{\xi_{o}^{\perp}}\right)^{-1}$. In this way, we get a function $W\left(t, \xi_{o}\right)$ defined on the 2 -sphere. 
Theorem 4.3. Let $W\left(t, \xi_{o}\right)$ be the solution of equation 4.31 with $W\left(0, \xi_{o}\right)=i d$, and suppose we have $\operatorname{Tr} W\left(t_{o}, \xi_{o}\right)=2$ for some $t_{o}>0$ and $\xi_{o} \in S^{2}$ with $\left\langle\xi_{o}, \omega_{o}\left(x_{o}\right)\right\rangle \neq 0$. Then we can solve the equation $\operatorname{Tr} W\left(t, \xi_{o}\right)=2$ for $t$ in terms of $\xi$, differentiably in any neighborhood of $\xi_{o}$.

Proof. Let us consider the 2 -sphere as a manifold; it is enough to prove that if $y$ is a coordinate on $S^{2}$ in some system, then $\frac{\partial t}{\partial y}$ exists. So let us suppose the other coordinate as fixed, and think of $W(t, y)$ as depending only on $y$. We assume $y=y_{o}$ corresponds to the specified point $\xi_{o}$ in $S^{2}$.

Since $\operatorname{det} W(t, y) \equiv 1$, we can show using the Cayley-Hamilton Theorem that we have $\left[W\left(t_{o}, y_{o}\right)-\mathrm{id}\right]^{2}=0$. Thus $W\left(t_{o}, y\right)-\mathrm{id}=N$ for some nilpotent matrix $N$, which must look like $N=c J \zeta \zeta^{T}$ for some unit vector $\zeta$ and real number $c$. Directly from equation 4.31, we have

$$
\begin{aligned}
\frac{\partial}{\partial t} \operatorname{Tr} W\left(t_{o}, y_{o}\right) & =-\operatorname{Tr}\left(J \Theta\left(t_{o}, y_{o}\right)\left(\mathrm{id}+c J \zeta \zeta^{T}\right)\right) \\
& =-c \operatorname{Tr}\left(J \Theta\left(t_{o}, y_{o}\right) J \zeta \zeta^{T}\right) \\
& =-c\left(\left\langle\zeta, J \Theta\left(t_{o}, y_{o}\right) J \zeta \zeta^{T}(\zeta)\right\rangle+\left\langle J \zeta, J \Theta\left(t_{o}, y_{o}\right) J \zeta \zeta^{T}(J \zeta)\right\rangle\right) \\
& =c\left\langle J \zeta, \Theta\left(t_{o}, y_{o}\right) J \zeta\right\rangle .
\end{aligned}
$$

In the first line, we used the familiar fact that if $A$ is antisymmetric and $B$ is symmetric, then $\operatorname{Tr} A B=0$. (We will use this again many times throughout the proof.) Now the term $\left\langle J \zeta, \Theta\left(t_{o}, y_{o}\right) J \zeta\right\rangle$ is positive since $\Theta$ is positive-definite, so that if $c \neq 0$, then $\frac{\partial \operatorname{Tr} W\left(t_{o}, y_{o}\right)}{\partial t} \neq 0$. As a result, we can solve for $t$ in terms of $y$ by the implicit function theorem (and hence obtain $\left.\frac{d t}{d y}\right)$ if $W\left(t_{o}, y_{o}\right) \neq \mathrm{id}$. On the other hand, if $W\left(t_{o}, y_{o}\right)=\mathrm{id}$, then $\frac{\partial \operatorname{Tr} W}{\partial t}\left(t_{o}, y_{o}\right)=0$. Thus we have to work a bit harder.

So now we suppose that $W\left(t_{o}, y_{o}\right)=\mathrm{id}$. Since the coefficients of 4.31) are smooth in $y$, we can differentiate $W(t, y)$ with respect to $y$. If we differentiate (4.31) with respect to $y$ and write $\frac{\partial W}{\partial y}=W M$, then $M$ must satisfy the equation

$$
\frac{\partial M}{\partial t}=-W^{-1} J \frac{\partial \Theta}{\partial y} W
$$

and since $\operatorname{Tr}\left(W^{-1} J \frac{\partial \Theta}{\partial y} W\right)=\operatorname{Tr}\left(J \frac{\partial \Theta}{\partial y}\right) \equiv 0$, we have $\frac{\partial}{\partial t} \operatorname{Tr} M(t, y) \equiv 0$ for all $t$ and $y$, so that $\operatorname{Tr} M(t, y)=0$ for all $t$ and $y$. Thus since $W\left(t_{o}, y_{o}\right)=\mathrm{id}$, we have $\frac{\partial \operatorname{Tr} W}{\partial y}\left(t_{o}, y_{o}\right)=$ $\operatorname{Tr} M\left(t_{o}, y_{o}\right)=0$.

So both partial derivatives of $\operatorname{Tr} W$ are zero at $\left(t_{o}, y_{o}\right)$, and thus we can look at second derivatives. If $\frac{d t}{d y}$ exists, then we must have (by differentiating $\operatorname{Tr} W(t(y), y)$ twice with respect to $y$ )

$$
\frac{\partial^{2} \operatorname{Tr} W}{\partial t^{2}}\left(t_{o}, y_{o}\right)\left(\frac{d t}{d y}\right)^{2}+2 \frac{\partial^{2} \operatorname{Tr} W}{\partial t \partial y}\left(t_{o}, y_{o}\right) \frac{d t}{d y}+\frac{\partial^{2} \operatorname{Tr} W}{\partial y^{2}}\left(t_{o}, y_{o}\right)=0
$$


and in order to be able to solve this for $\frac{d t}{d y}$, we must have

$$
\left(\frac{\partial^{2} \operatorname{Tr} W}{\partial t \partial y}\left(t_{o}, y_{o}\right)\right)^{2}-\frac{\partial^{2} \operatorname{Tr} W}{\partial t^{2}}\left(t_{o}, y_{o}\right) \frac{\partial^{2} \operatorname{Tr} W}{\partial y^{2}}\left(t_{o}, y_{o}\right) \geq 0
$$

Conversely if this condition is satisfied, we obtain either one or two values for $\frac{d t}{d y}$, corresponding to a crossing of at most two solutions.

Observe first that, quite generally, if $W$ is a matrix with $\operatorname{det} W \equiv 1$, then for any parameter $r$ we have $\operatorname{Tr}\left(W^{-1} \frac{\partial W}{\partial r}\right)=0$. If we then differentiate with respect to any other parameter $s$, we have

$$
\operatorname{Tr}\left(W^{-1} \frac{\partial^{2} W}{\partial r \partial s}\right)=\operatorname{Tr}\left(W^{-1} \frac{\partial W}{\partial r} W^{-1} \frac{\partial W}{\partial s}\right) .
$$

Now in our special case, we have $W\left(t_{o}, y_{o}\right)=$ id so that

$$
\frac{\partial^{2} \operatorname{Tr} W}{\partial \theta \partial t}\left(t_{o}, y_{o}\right)=\operatorname{Tr}\left(\frac{\partial W}{\partial t}\left(t_{o}, y_{o}\right) \frac{\partial W}{\partial y}\left(t_{o}, y_{o}\right)\right)
$$

while

$$
\frac{\partial^{2} \operatorname{Tr} W}{\partial y^{2}}\left(t_{o}, y_{o}\right)=\operatorname{Tr}\left(\frac{\partial W}{\partial y}\left(t_{o}, y_{o}\right)\right)^{2}=-2 \operatorname{det}\left(\frac{\partial W}{\partial y}\left(t_{o}, y_{o}\right)\right)
$$

and

$$
\frac{\partial^{2} \operatorname{Tr} W}{\partial t^{2}}\left(t_{o}, y_{o}\right)=\operatorname{Tr}\left(\frac{\partial W}{\partial t}\left(t_{o}, y_{o}\right)\right)^{2}=-2 \operatorname{det}\left(\frac{\partial W}{\partial t}\left(t_{o}, y_{o}\right)\right)
$$

(these last two are consequences of the Cayley-Hamilton theorem).

Thus the inequality 4.33 translates into

$$
\left[\operatorname{Tr}\left(\frac{\partial W}{\partial t} \frac{\partial W}{\partial y}\right)\right]^{2}-4 \operatorname{det}\left(\frac{\partial W}{\partial t} \frac{\partial W}{\partial y}\right) \geq 0 \quad \text { at }\left(t_{o}, y_{o}\right)
$$

Now the equation $\operatorname{Tr} A^{2}-4 \operatorname{det} A \geq 0$ for a $2 \times 2$ matrix $A$ is equivalent to the requirement that $A$ has real eigenvalues. Thus we will have (4.33) if

$$
\frac{\partial W}{\partial t}\left(t_{o}, y_{o}\right) \frac{\partial W}{\partial y}\left(t_{o}, y_{o}\right)=J \Theta\left(t_{o}, y_{o}\right) \int_{0}^{t} W\left(\tau, y_{o}\right)^{-1} J \frac{\partial \Theta}{\partial y}\left(\tau, y_{o}\right) W\left(\tau, y_{o}\right) d \tau
$$

has real eigenvalues. To establish this, we eliminate the $J$ matrices using the easilyproved formula $J Z=(\operatorname{det} Z)\left(Z^{T}\right)^{-1} J$ along with $J^{2}=-$ id to obtain

$$
\frac{\partial W}{\partial t}\left(t_{o}, y_{o}\right) \frac{\partial W}{\partial y}\left(t_{o}, y_{o}\right)=\operatorname{det}\left(\Theta\left(t_{o}, y_{o}\right)\right) \Theta\left(t_{o}, y_{o}\right)^{-1} \int_{0}^{t_{o}} W\left(\tau, y_{o}\right)^{T} \frac{\partial \Theta}{\partial y}\left(t_{o}, y_{o}\right) W\left(\tau, y_{o}\right) d \tau \text {. }
$$

We now have the desired matrix as a product of a symmetric positive-definite matrix and a symmetric matrix. It is easy to see that such a product must have real eigenvalues: let $C$ be a symmetric positive-definite matrix and $D$ a symmetric matrix. In an eigenvector-basis of $C$, we have $C=\left(\begin{array}{ll}\lambda & 0 \\ 0 & \mu\end{array}\right)$ and $D=\left(\begin{array}{ll}a & b \\ b & c\end{array}\right)$, so that $C D=\left(\begin{array}{ll}\lambda a & \lambda b \\ \mu b & \mu c\end{array}\right)$. The expression $[\operatorname{Tr}(C D)]^{2}-4 \operatorname{det}(C D)$ becomes $(\lambda a-\mu c)^{2}+4 \lambda \mu b^{2}$, and this is nonnegative since $\lambda \mu>0$. So $C D$ must have real eigenvalues, and this establishes (4.33). 
Corollary 4.4. For each point $x_{o} \in M$, there is a family of $C^{1}$ functions $f\left(\xi_{o}\right)$ defined on open subsets of the 2-sphere, such that for every $\xi_{\text {o }}$ there is a weakly epiconjugate point to the identity located at $t=f\left(\xi_{o}\right)$. As a result, the set of all weakly epiconjugate point locations obtainable by the technique of Theorem 3.4 consists of the closure of the union of open intervals.

Proof. For every component of the domain of $f$, the image of $f$. So we have a family of intervals (possibly open, closed, or half-open) as $\xi_{o}$ ranges over $S^{2}$. The actual set of epiconjugate points obtained this way must be closed, since if we have an sequence of conjugate point locations then any limit point must be an epiconjugate point location. Hence we obtain some union of closed intervals.

By Theorem 2.5 and Theorem 2.7, we can conclude the following about the intervals obtained from Corollary 4.4 .

Corollary 4.5. All of the points in the nontrivial intervals obtained by Corollary 4.4 are strongly epiconjugate.

Proof. Take any nontrivial closed interval of weakly epiconjugate point locations $[a, b] \subset$ $\mathbb{R}^{+}$. By Theorem 2.5, there is a countable dense subset of this interval consisting of weakly monoconjugate points. All other points in the interval must therefore be strictly weakly epiconjugate, and thus strongly epiconjugate. Finally, since a limit of strongly epiconjugate points is also strongly epiconjugate, the entire interval must consist of strongly epiconjugate points.

It is not clear whether this technique actually generates more than one interval; in the simple examples one can work out explicitly, there is always just a single interval extending to infinity. The following is a simple yet seemingly typical example.

Proposition 4.6. Suppose $u$ is a Killing field on $M$. (Such a $u$ is a steady solution of the Euler equation, by Misiotek [?].) The epiconjugate points along the corresponding geodesic $\eta$ form an interval $[\tau, \infty)$ for some $\tau>0$.

Proof. Since $u$ is a Killing field, the metric pullback $\Lambda(t, x)$ must be the identity for all $t$ and $x$. Thus the equation (3.24) takes the form

$$
\frac{d^{2} \gamma}{d t^{2}}+\left\langle\omega_{o}\left(x_{o}\right), \xi_{o}\right\rangle \xi_{o} \times \frac{d \gamma}{d t}=0
$$

with $\gamma(t)$ orthogonal to $\xi_{o}$ for all $t$. It is easy to see that this equation has solutions vanishing at times

$$
\tau\left(x_{o}, \xi_{o}\right)=\frac{2 \pi}{\left\langle\omega_{o}\left(x_{o}\right), \xi_{o}\right\rangle}
$$

for any unit vector $\xi_{o} \in T_{x_{o}} M$. The infimum $\tau\left(x_{o}\right)=\inf _{\xi_{o} \in S^{2}} \tau\left(x_{o}, \xi_{o}\right)$ occurs when $\xi_{o}$ is parallel to $\omega_{o}\left(x_{o}\right)$ and is $\tau\left(x_{o}\right)=\frac{2 \pi}{\left|\omega_{o}\left(x_{o}\right)\right|}$. The absolute minimum over all $x_{o} \in M$ is

$$
\tau=\inf _{x_{o} \in M} \tau\left(x_{o}\right)=\frac{2 \pi}{\left\|\omega_{o}\right\|_{L^{\infty}}} .
$$


On the other hand, as $\xi_{o}$ approaches a vector orthogonal to $\omega_{o}\left(x_{o}\right)$, the time $\tau\left(x_{o}, \xi_{o}\right)$ obviously approaches infinity for any $x_{o}$.

Now it is also not hard to see that the actual first conjugate point occurs at $\tau=$ $\frac{2 \pi}{\left\|\omega_{o}\right\|_{L^{\infty}}}$ (this is proved in [?]). As a result, in this case Theorem 3.4 predicts all conjugate point locations.

The behavior seen above for Killing fields appears to be typical; for the flows whose conjugate points one can work out explicitly, epiconjugate points occur in intervals extending to infinity. It is conceivable that for nonsteady flows, the metric pullback $\Lambda$ may increase fast enough with time to prevent solutions of (3.24) from vanishing at large times. Obviously, if the solution of the Euler equation blows up at a finite time, then all bets are off.

Department of Mathematics, University of Colorado, Boulder, CO 80309-0395

E-mail address: Stephen.Preston@colorado.edu 